\title{
Spontaneous emission enhancement of colloidal perovskite nanocrystals by a photonic crystal cavity
}

\author{
Zhili Yang, ${ }^{1}$ Matthew Pelton, ${ }^{2}$ Maryna I. Bodnarchuk,, ${ }^{3,4}$ Maksym V. Kovalenko, ${ }^{3,4}$ \\ and Edo Waks ${ }^{1,5, a)}$ \\ ${ }^{1}$ Department of Electrical Engineering and Institute for Research in Electronics and Applied Physics, \\ University of Maryland, College Park, Maryland 20742, USA \\ ${ }^{2}$ Department of Physics, University of Maryland, Baltimore County, Baltimore, Maryland 21250, USA \\ ${ }^{3}$ Institute of Inorganic Chemistry, Department of Chemistry and Applied Biosciences, ETH Zürich, \\ Vladimir Prelog Weg 1, CH-8093 Zürich, Switzerland \\ ${ }^{4}$ Laboratory for Thin Films and Photovoltaics, Empa-Swiss Federal Laboratories for Materials Science and \\ Technology, Überlandstrasse 129, CH-8600 Dübendorf, Switzerland \\ ${ }^{5}$ Joint Quantum Institute, University of Maryland and National Institute of Standards and Technology, \\ College Park, Maryland 20742, USA
}

(Received 14 August 2017; accepted 3 November 2017; published online 28 November 2017)

\begin{abstract}
We demonstrate coupling of lead halide perovskite nanocrystals to a nanophotonic cavity. From photoluminescence measurements, we observe a factor of 10 enhancement in brightness from the cavity mode emission. We perform room temperature time-resolved lifetime measurements that demonstrate an average spontaneous emission rate enhancement of 2.9 for perovskite nanocrystals within the cavity as compared to those located on the unpatterned surfaces. Our method provides a way towards realizing efficient light emitters and low-threshold lasers, as well as fast nonlinear optical devices, using solution processable materials. Published by AIP Publishing.

https://doi.org/10.1063/1.5000248
\end{abstract}

Solution-processable lead trihalide perovskite semiconductors are alternatives in optoelectronic applications to epitaxial materials that require complex growth capabilities to synthesize. ${ }^{1-4}$ Perovskites exhibit a small Stokes shift, ${ }^{5}$ high charge carrier mobility, ${ }^{6}$ and large absorption coefficient. ${ }^{7}$ These features could enable high-performance photovoltaic devices, light-emitting diodes, and light sources for efficient lasers. Previous studies focusing on photovoltaic applications adopted perovskite thin films as absorbing layers in solar cells with a record-high certified conversion efficiency of $22.1 \%{ }^{8}$ Beyond photovoltaics, perovskites could also serve as a new type of efficient emitter and gain material for opto-electronic applications. $^{9}$

Lead trihalide perovskite nanocrystals exhibit a number of properties that make them promising candidates for lightemitting devices. They exhibit slow nonradiative decay pathways, which reduces Auger recombination, a problem that many quantum emitters suffer from. ${ }^{10}$ Their exciton energy is widely tunable by chemical composition and quantum confinement. ${ }^{11}$ This allows the emission to cover the entire visible spectrum, especially the purple and blue wavelengths where other colloidal quantum emitters are hard to synthesize and emit poorly. In addition, lead trihalide perovskites exhibit low densities of carrier-trapping defects, which leads to high photoluminescence efficiency at room temperature. ${ }^{12-14}$ These beneficial properties enabled perovskitebased light-emitting diodes ${ }^{15,16}$ and lasers. ${ }^{17,18}$ More recent work realized enhanced lasing in perovskite nanocrystals coupled to band edge states of large area photonic crystal structures. ${ }^{19}$ Coupling these emitters to small mode-volume nanophotonic cavities could further improve the efficiency

\footnotetext{
a) Author to whom correspondence should be addressed: edowaks@umd.edu
}

of these emitters and also enable low-threshold lasers. ${ }^{20}$ Furthermore, the increased binding energies and oscillator strengths of perovskites make them a promising system to study strong light-matter interactions and cavity quantum electrodynamics. ${ }^{21}$ However, the integration of lead-halide perovskite nanocrystals with nanophotonic cavities remains unexplored.

In this paper, we demonstrate enhanced radiative emission of perovskite nanocrystals by coupling them to a silicon nitride photonic crystal nanocavity at room temperature. We show a significant brightness enhancement of the nanocrystals, along with an enhanced spontaneous emission rate with an average enhancement factor of 2.9. Our results provide a path toward compact on-chip light sources with reduced energy consumption and size.

Our device uses $\mathrm{CsPbBr}_{3}$ perovskite nanocrystals. We synthesize the $\mathrm{CsPbBr}_{3}$ nanocrystals in a crude solution and then centrifuge using the washing procedure described in our recent report. ${ }^{11}$ Figure 1 (a) presents the absorption and emission spectra of perovskite in toluene solution used in our experiment. We collect the absorption spectrum using a Jasco V670 spectrometer in the transmission mode and the photoluminescence spectrum using a Fluorolog iHR320 Horiba Jobin Yvon spectrofluorometer equipped with a photomultiplier detector. The excitation wavelength is $400 \mathrm{~nm}$, provided by a $450 \mathrm{~W}$ Xenon lamp dispersed with a monochromator. Measured intensities are corrected to take into account the spectral response of the detector.

They exhibit photoluminescence centered at $510 \mathrm{~nm}$ with a full-width at half-maximum linewidth of $20 \mathrm{~nm}$, and the corresponding absorption onset is at $501 \mathrm{~nm}$. Figure 1(b) displays a transmission electron microscopy image of the ensemble nanocrystals after depositing the solution on the 
(a)

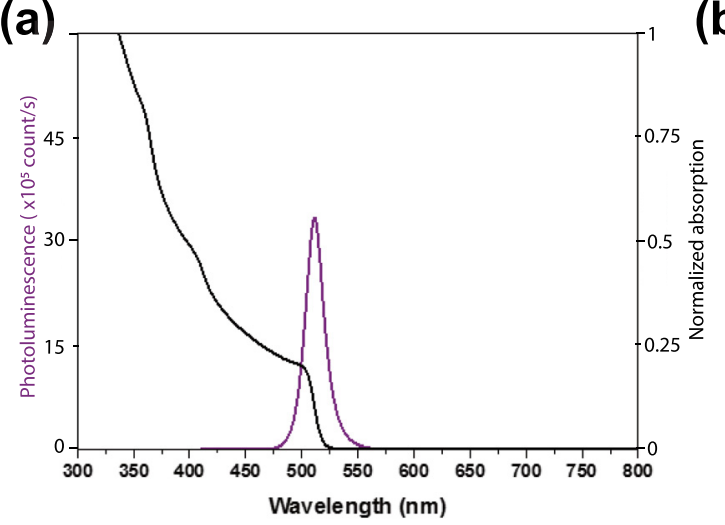

(b)

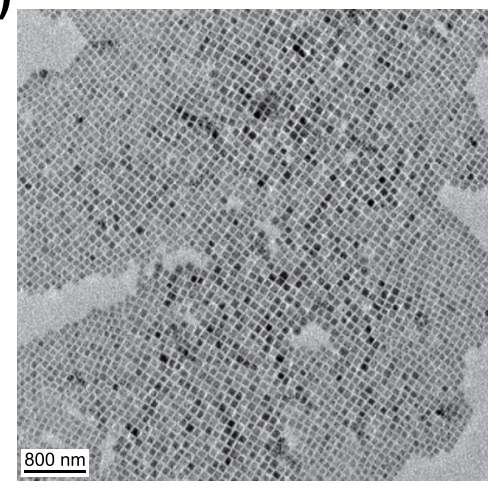

FIG. 1. (a) Absorption (black) and photoluminescence (purple) spectra of perovskite nanocrystals in solution form. (b) TEM image of perovskite nanocrystals deposited on the sample surface. sample surface using the drop-casting method. The nanocrystals are cubes with side lengths on the order of $10 \mathrm{~nm}$, and the formed film on the sample surface is uniform across a large area.

We couple the perovskite nanocrystals to a silicon nitride photonic crystal nanobeam cavity designed with a cavity resonance at $510 \mathrm{~nm},{ }^{22}$ which is matched to the peak photoluminescence emission of the perovskites. We adapted the cavity design proposed by Khan et al. for our specific wavelength. ${ }^{22}$ The cavity design consists of a 200-nm-thick and 270-nmwide $\mathrm{SiN}$ beam with a one-dimensional periodic array of air holes with a lattice constant of $180 \mathrm{~nm}$ and a hole radius of $60 \mathrm{~nm}$. We form the cavity by linearly reducing both the lattice constant and the radius of the four holes at the center of the beam on both sides. We reduce the lattice constant from $180 \mathrm{~nm}$ to $153 \mathrm{~nm}$, and the hole radius from $60 \mathrm{~nm}$ to $45 \mathrm{~nm}$. Figure 2(a) displays the calculated mode profile of the designed cavity structure obtained from a finite-difference time-domain simulation. The design achieves a resonant wavelength of $510 \mathrm{~nm}$ and a calculated quality factor of 35000 .

To fabricate the device, we begin with an initial wafer composed of $200 \mathrm{~nm}$ thick stoichiometric SiN deposited on a $\mathrm{Si}$ substrate. We grow the $\mathrm{SiN}$ layer using low-pressure chemical vapor deposition. We then use electron beam lithography followed by fluorine-based inductively coupled plasma dry etching to fabricate the photonic crystal. We wetetch the underlying silicon using aqueous $\mathrm{KOH}$ to create the suspended nanobeam cavity structure shown in the scanning electron microscopy image in Fig. 2(b). After fabrication, we deposit perovskite nanocrystals onto the cavities by dropcasting a toluene solution $(0.1 \mathrm{mg} / \mathrm{mL})$ onto the sample surface.

Figure 2(c) illustrates the experimental setup for investigating our coupled device. We perform the measurement on the device using a lab-built confocal microscopy system. We excite and collect the signal from the sample using an objective lens with a numerical aperture of 0.6. We pump the sample with 70-ps duration laser pulses with a repetition rate of $20 \mathrm{MHz}$ at a wavelength of $405 \mathrm{~nm}$. We filter out the collected signal spectrally and spatially using a 450-nm, longpass filter and an iris, respectively. We then focus the signal onto a grating spectrometer for spectral analysis. To measure the lifetime, we spectrally filter out the cavity signal using a spectrometer with a resolution of $0.1 \mathrm{~nm}$ and measure it using an avalanche photodiode single-photon counter with a timing resolution of $220 \mathrm{ps}$. We use a time interval analyzer to correlate the arriving photon events with the pump laser pulses to obtain a lifetime trace.

Figure 3(a) shows the emission spectrum of one device, as well as that from an unpatterned region of the sample (a)

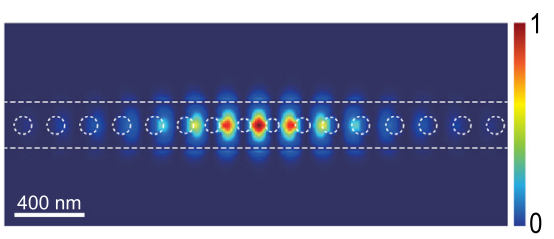

(c)

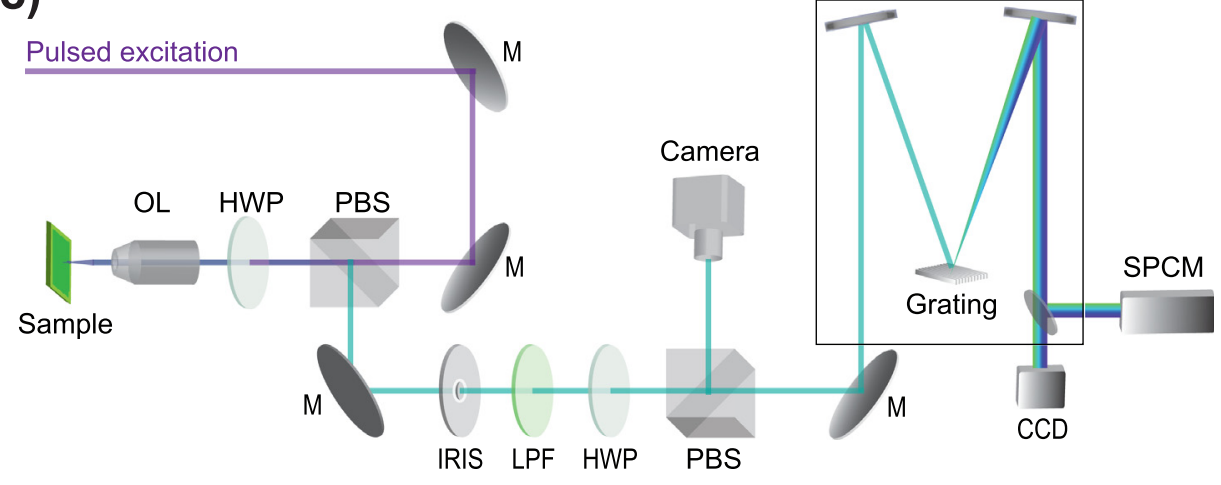

FIG. 2. (a) Calculated electric field intensity profile of the fundamental mode in the cavity. (b) Scanning electron microscopy image of a SiN photonic crystal nanobeam cavity. (c) Experimental setup. M, mirror; PBS, polarizing beam splitter; HWP, halfwave plate; OL, objective lens; LPF, long-pass filter (cutoff at $450 \mathrm{~nm}$ ); IRIS, iris as a spatial filter; SPCM, single-photon counting module; CCD, charge-coupled device. 

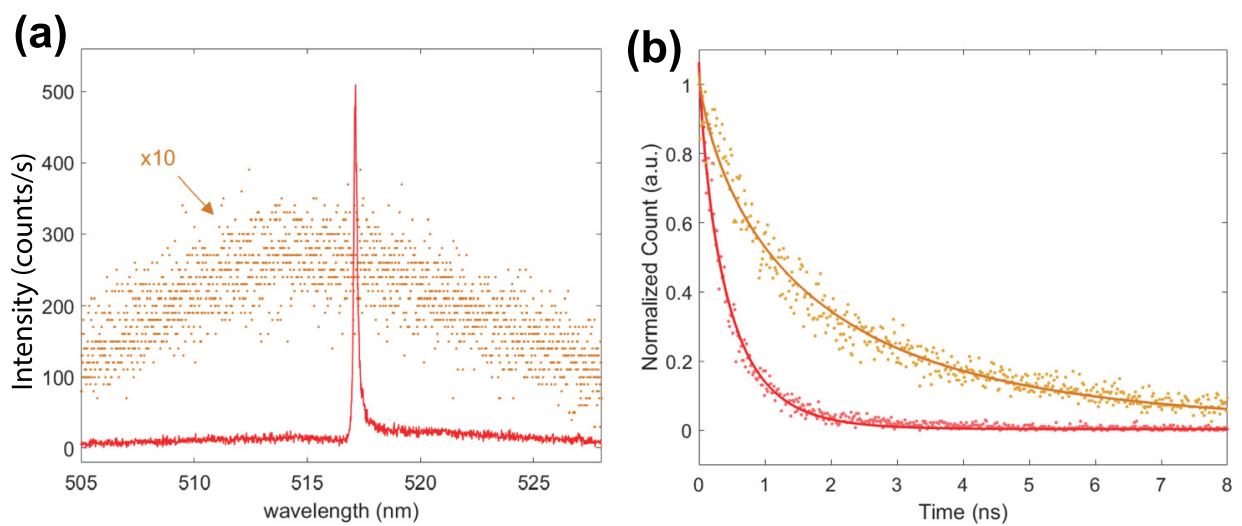

FIG. 3. (a) Emission spectra from perovskites on the cavity (red) and in the unpatterned region (yellow, multiplied by a factor of 10 for clarity), both measured under pulsed excitation. (b) Normalized time-resolved photoluminescence from perovskites on the cavity (red) and in an unpatterned region (yellow). The dots indicate the measured data, and the lines are the fits to the stretched exponential decay model.

(multiplied by a factor of 10) with an excitation power of $1 \mu \mathrm{W}$. We measure a laser spot size of $0.45 \mu \mathrm{m}$ in radius, leading to a pump power density of $150 \mathrm{~W} / \mu \mathrm{m}^{2}$. The photoluminescence spectrum of the perovskites in the unpatterned region has a full-width at half-maximum linewidth of $20 \mathrm{~nm}$. In contrast, those coupled to the cavity mode exhibit a sharp peak emission at the cavity resonant frequency, where the signal is more than 10 times brighter than that of the unpatterned region. By fitting to a Lorentzian function, we obtain a Q factor of $4000 \pm 300$ for this coupled device. We note that the nanobeam cavity possesses a much smaller surface area compared to the unpatterned region. Despite the fact that we pump a smaller number of nanocrystals, we still observe a much larger signal.

We next measure radiative lifetimes of the emission from the perovskite nanocrystals. Figure 3(b) shows the timeresolved lifetime measurements at the cavity and on the unpatterned surface. In both cases, the decay is multi-exponential. ${ }^{23}$ Perovskite ensembles typically exhibit a multi-exponential decay due to variations in the non-radiative decay rate. ${ }^{24}$ Perovskites coupled to the cavity may exhibit additional fluctuations in the radiative decay rate because they are in different positions within the cavity and therefore experience different radiative enhancements. To account for the variations in the decay rate, we fit the decay to a stretched exponential function of the form

$$
I(t)=I_{0}+A e^{-\left(t / \tau_{s e}\right)^{\beta}}
$$

where $I(t)$ is the photoluminescence intensity at time $t, I_{O}$ is the background intensity, $A$ is a scaling constant, $1 / \tau_{s e}$ is the decay constant of excitons in perovskites, and $\beta$ is the stretch parameter which ranges from 0 to 1 . This parameter indicates the variance in the decay rates. The higher the value of the stretch parameter, the lower the variance, with $\beta=1$ corresponding to a single-exponential decay. From the stretched exponential fit, we calculate the ensemble average lifetime using the following expression: ${ }^{23}$

$$
T_{a v g}=\frac{T_{s e}}{\beta} \Gamma\left(\frac{1}{\beta}\right),
$$

where $\Gamma(z)=\int_{0}^{\infty} x^{z-1} e^{-x} d x$ is the Gamma function.

We performed lifetime measurements on 10 different devices. For each device, we also measured the unpatterned surface nearby as a comparison. The average lifetime of the perovskites on the unpatterned surface is $1.36 \pm 0.50 \mathrm{~ns}$ with $\beta=0.74$. In contrast, the cavity-coupled perovskites exhibit a shorter average lifetime of $0.46 \pm 0.05 \mathrm{~ns}$ with $\beta=0.82$. We define the Purcell factor as the ratio of the average cavity lifetime to the average lifetime of the unpatterned surface near the cavity. From the 10 measurements, we attain an average Purcell factor of $2.9 \pm 0.9$, confirming the enhancement of spontaneous emission.

We compare the measured lifetime with the theoretical calculation by the FDTD simulation using a model in the bad emitter regime ${ }^{25}$

$$
F_{\max }=1+\frac{3 \lambda^{3}}{4 \pi^{2} n^{2}} \frac{Q_{\mathrm{p}}}{V} \psi(\mathbf{r}) .
$$

This expression denotes the Purcell factor for an emitter located on the nanobeam surface at the maximum field intensity position. Here, $\lambda$ is the cavity mode wavelength, $Q_{\mathrm{p}}$ $=\lambda / \Delta \lambda_{\mathrm{p}}$ is the quality factor of the perovskite emission where the linewidth is $\Delta \lambda_{\mathrm{p}}, n$ is the refractive index of $\mathrm{SiN}$ at the cavity resonance, $V=\int d^{3} \mathbf{r} \varepsilon(\mathbf{r})|E(\mathbf{r})|^{2} /\left[\varepsilon(\mathbf{r})|E(\mathbf{r})|^{2}\right]_{\max }$ is the cavity mode volume, $\varepsilon(\mathbf{r})$ is the relative dielectric constant, and $\psi(\mathbf{r})=|E(\mathbf{r})|^{2} /|E(\mathbf{r})|_{\max }^{2}$ is the ratio of the cavity field intensity at position $r$ to that at its maximum located in the center of the beam. We note that our system operates in the bad emitter regime, where the linewidth of the emitter is much broader than the cavity linewidth. In this limit, the Purcell factor is independent of the cavity quality factor and depends only on the emitter linewidth. ${ }^{25}$ From the FDTD simulations, we obtain $V=0.55(\lambda / n)^{3}$ and $\psi(\mathbf{r})=0.36$ for an emitter located at the center of the nanobeam cavity surface. Substituting these values into Eq. (3), we obtain a maximum Purcell factor of 3.6, which agrees well with the measured value. We attribute the lower Purcell factors measured in the actual devices to the fact that we are exciting an area of perovskites that are not all located at the field maximum on the nanobeam surface.

In conclusion, we demonstrated the enhancement in the brightness and radiative decay rate of trihalide perovskite nanocrystals by coupling to a one-dimensional photonic crystal nanocavity at room temperature. We obtained an enhancement factor of 2.9 in the average spontaneous emission rate. We could further increase the enhancement by designing cavities confining the mode in the air so that the nanocrystals will be in the region with the maximum cavity field. ${ }^{26}$ By controlling the size and material composition, we can create 
enhanced light emitters spanning the entire visible range, especially for the blue-green wavelengths which are previously hard to achieve. ${ }^{11}$ Our method could also improve the efficiency of existing perovskite LEDs to open up real world applications such as efficient illumination and displays. ${ }^{27,28}$ Finally, our results could enable lasers with low thresholds and nonlinear optical devices with fast responses, which are crucial components in integrated optoelectronics.

${ }^{1}$ B. R. Sutherland and E. H. Sargent, Nat. Photonics 10(5), 295-302 (2016). ${ }^{2}$ H.-S. Kim, S. H. Im, and N.-G. Park, J. Phys. Chem. C 118(11), 5615-5625 (2014).

${ }^{3}$ S. D. Stranks and H. J. Snaith, Nat. Nanotechnol. 10(5), 391-402 (2015).

${ }^{4}$ M. A. Green, A. Ho-Baillie, and H. J. Snaith, Nat. Photonics 8(7), 506-514 (2014).

${ }^{5}$ S. D. Stranks, G. E. Eperon, G. Grancini, C. Menelaou, M. J. Alcocer, T. Leijtens, L. M. Herz, A. Petrozza, and H. J. Snaith, Science 342(6156), 341-344 (2013).

${ }^{6}$ T. Leijtens, S. D. Stranks, G. E. Eperon, R. Lindblad, E. M. Johansson, I. J. McPherson, H. Rensmo, J. M. Ball, M. M. Lee, and H. J. Snaith, ACS Nano 8(7), 7147-7155 (2014).

${ }^{7}$ S. De Wolf, J. Holovsky, S.-J. Moon, P. Löper, B. Niesen, M. Ledinsky, F.-J. Haug, J.-H. Yum, and C. Ballif, J. Phys. Chem. Lett. 5(6), 1035-1039 (2014).

${ }^{8}$ http://www.nrel.gov/ncpv for Research Cell Efficiency Records, National Renewable Energy Laboratory; accessed 1 April 2016.

${ }^{9}$ Y. Wang, X. Li, J. Song, L. Xiao, H. Zeng, and H. Sun, Adv. Mater. 27(44), 7101-7108 (2015).

${ }^{10}$ F. Deschler, M. Price, S. Pathak, L. E. Klintberg, D.-D. Jarausch, R. Higler, S. Huittner, T. Leijtens, S. D. Stranks, and H. J. Snaith, J. Phys. Chem. Lett. 5(8), 1421-1426 (2014).

${ }^{11}$ L. Protesescu, S. Yakunin, M. I. Bodnarchuk, F. Krieg, R. Caputo, C. H. Hendon, R. X. Yang, A. Walsh, and M. V. Kovalenko, Nano Lett. 15(6), 3692-3696 (2015).
${ }^{12}$ R. E. Brandt, V. Stevanović, D. S. Ginley, and T. Buonassisi, MRS Commun. 5(02), 265-275 (2015).

${ }^{13}$ J. Kang and L.-W. Wang, J. Phys. Chem. Lett. 8, 489-493 (2017).

${ }^{14}$ S. Ten Brinck and I. Infante, ACS Energy Lett. 1(6), 1266-1272 (2016).

${ }^{15}$ J. Wang, N. Wang, Y. Jin, J. Si, Z. K. Tan, H. Du, L. Cheng, X. Dai, S. Bai, and H. He, Adv. Mater. 27(14), 2311-2316 (2015).

${ }^{16}$ H. Cho, S.-H. Jeong, M.-H. Park, Y.-H. Kim, C. Wolf, C.-L. Lee, J. H. Heo, A. Sadhanala, N. Myoung, and S. Yoo, Science 350(6265), 1222-1225 (2015).

${ }^{17}$ S. Yakunin, L. Protesescu, F. Krieg, M. I. Bodnarchuk, G. Nedelcu, M. Humer, G. D. Luca, M. Fiebig, W. Heiss, and M. V. Kovalenko, Nat. Commun. 6, 8056 (2015).

${ }^{18}$ H. Zhu, Y. Fu, F. Meng, X. Wu, Z. Gong, Q. Ding, M. V. Gustafsson, M. T. Trinh, S. Jin, and X. Zhu, Nat. materials 14(6), 636-642 (2015).

${ }^{19}$ S. Chen, K. Roh, J. Lee, W. K. Chong, Y. Lu, N. Mathews, T. C. Sum, and A. Nurmikko, ACS Nano 10(4), 3959-3967 (2016).

${ }^{20}$ M. Lončar, T. Yoshie, A. Scherer, P. Gogna, and Y. Qiu, Appl. Phys. Lett. 81(15), 2680-2682 (2002).

${ }^{21}$ L. N. Quan, M. Yuan, R. Comin, O. Voznyy, E. M. Beauregard, S. Hoogland, A. Buin, A. R. Kirmani, K. Zhao, and A. Amassian, J. Am. Chem. Soc. 138(8), 2649-2655 (2016).

${ }^{22}$ M. Khan, T. Babinec, M. W. McCutcheon, P. Deotare, and M. Lončar, Opt. Lett. 36(3), 421-423 (2011).

${ }^{23}$ B. R. Fisher, H.-J. Eisler, N. E. Stott, and M. G. Bawendi, J. Phys. Chem. B 108(1), 143-148 (2004).

${ }^{24}$ Y.-S. Park, S. Guo, N. S. Makarov, and V. I. Klimov, ACS Nano 9(10), 10386-10393 (2015).

${ }^{25}$ M. Van Exter, G. Nienhuis, and J. Woerdman, Phys. Rev. A 54(4), 3553 (1996).

${ }^{26}$ Q. Quan and M. Loncar, Opt. Express 19(19), 18529-18542 (2011).

${ }^{27}$ Z. Xiao, R. A. Kerner, L. Zhao, N. L. Tran, K. M. Lee, T.-W. Koh, G. D. Scholes, and B. P. Rand, Nat. Photonics 11(2), 108-115 (2017).

${ }^{28}$ N. Wang, L. Cheng, R. Ge, S. Zhang, Y. Miao, W. Zou, C. Yi, Y. Sun, Y. Cao, and R. Yang, Nat. Photonics 10(11), 699-704 (2016). 